\title{
ON POSSIBILITIES OF FAST COOLING OF HEAVY PARTICLE BEAMS
}

\author{
Ya. S. Derbenev \\ Randall Laboratory of Physics, University of Michigan \\ Ann Arbor, Michigan 48109-1120 USA
}

\begin{abstract}
Two methods of fast cooling of intensive beams are described. The first one, coherent electron cooling, is based on enhancement of friction effect in the electron cooling method using a microwave instability of electron beam specially arranged in the cooling section. This method is effective for cooling of hightemperature circulating beams. The second one, self-cooling, is based on use of the intrabeam Coulomb scattering of particles during the adiabatic processes of beam acceleration and transverse compression. This method allows frequent decrease emittance of an intensive beam issued by a low-temperature source.
\end{abstract}

\section{Coherent electron Cooling}

Three methods of cooling of circulating heavy particle beams are known today: electron cooling, which is based on the use of a co-moving electron beam, straight or circulating, as in a thermostat ${ }^{(1-3)}$; stochastic cooling based on the use of RF feedback system ${ }^{[4,5]}$; laser cooling of ion beams ${ }^{[6]}$. Electron and laser cooling are efficient to cool low-temperature intensive beam; oppositely, stochastic cooling is effective to cool high-temperature, low-intensity beams. The coherent electron cooling ${ }^{[7,8]}$ described below combines principles and advantages of both electron and stochastic cooling.

First, let us give the qualitative consideration in favor of the principle possibility to increase the friction effect at Coulomb interaction. As it is known, a fast charged particle in plasma is effected by the friction force

$$
\vec{F}(\vec{v})=-\frac{4 \pi Z^{2} e^{4} n_{e}}{m v^{2}} L(v) \frac{\vec{v}}{v} \equiv-\frac{(Z e)^{2}}{\rho_{s h}^{2}} L(v) \frac{\vec{v}}{v}
$$

where $\mathrm{Ze}$ is a particle charge, $\mathrm{e}, \mathrm{m}$ and $\mathrm{n}_{\mathrm{e}}$ are respectively the charge, mass and density of electrons, $\mathrm{L}(\mathrm{v})=\ln \left(\rho_{\max } / \rho_{\min }\right)$ is the Coulomb logarithm. The fast condition means that the particle velocity $\mathrm{v}$ is large compared to the electron heat velocity $\mathrm{v}_{\mathrm{e}}=\sqrt{\mathrm{T}_{\mathrm{e}} / \mathrm{m}}$. In this case the maximum impact parameter in Coulomb logarithm is equal to the distance of dynamical shielding for effecting Coulomb forces: $\rho_{\max }=\rho_{\mathrm{sh}}=\mathrm{v} / \omega_{\mathrm{e}}$ where $\omega_{\mathrm{e}}=\left(4 \pi \mathrm{n}_{\mathrm{e}} \mathrm{e}^{2} / \mathrm{m}\right)^{1 / 2}$ is an electron plasma frequency. Since the particle is fast, this distance is large compared to the 
Debay radius of electron gas: $\rho_{\mathrm{sh}} \gg \mathrm{rD}_{\mathrm{D}}=\mathrm{v}_{\mathrm{e}} / \omega_{\mathrm{e}}$ which means that the plasma collective degrees of freedom corresponding to excitation in a characteristic time $\omega_{\mathrm{e}}^{-1}$ of a group electron motion in the range of a radius $\sim \rho_{s h}$ are effectively participate in interaction with fast particle. This excitation, however, is so small that the contribution of collective modes into the total response is reduced to an insignificant increase in the Coulomb logarithm $\left(\rho_{\text {min }} \sim \mathrm{Ze}^{2} / \mathrm{mv}^{2}\right)$; effectively, a fast particle interacts with its image on a distance about $\rho_{\mathrm{sh}}$.

The collective response could be increased proportionally to the number of electrons in the interaction region if the initial excitations could increase spontaneously. For this, the electron plasma should be able to self-bunching, i.e. should be unstable in the region of the wave-lengths $\lambda>\mathrm{r}_{\mathrm{D}}$.

A principle amplification can be naturally inserted into the scheme of the electron cooling method. On the cooling section such conditions should be arranged that the moving "electron plasma" should become unstable in the given range of the wave lengths. Then, an excitation caused by an input ion will be transferred by electron flux developing exponentially independent of the ion; at the output from electron beam the ion will acquire the momentum correlated with its input velocity. In any case, quite a strong correlation is possible unless the excitation reaches the nonlinear regime, i.e. the density modulation within the required scale of distances remains relatively small. It is, of course, necessary to provide the optimum output phase relations in the position, and velocity of an ion with respect to electron "avalanche" produced by the ion. Such a task is facilitated by the motion of ions and electrons in the fields given is absolutely different. In particular, after interaction at the "input" the beams can be separated and then they can be made interacting again at the "output".

The mechanism of instability with the properties required can be precluded if in the cooling section the transverse oscillating with period $\lambda_{0}$ along the beam path magnetic field of relatively small amplitude is introduced into the longitudinal magnetic field accompanying an electron flux with Larmour period $\lambda_{\mathrm{L}}$.

In such a system, which is called an undulator, three type of instabilities $(\mathrm{a}, \mathrm{b}, \mathrm{c})$ are possible ${ }^{[9-12]}$ :

a. An instability of the negative longitudinal mass can be realized in the region $\lambda_{\mathrm{L}}<\lambda_{0}$ when an average velocity becomes a decreasing function of an energy because of an increase in the forced transverse velocity.

This kind of instability can be easily arranged at relativistic energies.

b. Near the point $\lambda_{\mathrm{L}}=\lambda_{0}$ another kind of Coulomb instability occurs which can be called the cyclotron-ondulator instability. The use of this instability seems most preferable for non-relativistic case.

c. In relativistic region the mechanism of radiative instability can also be effective which is connected to generation of coherent radiation. The systems 
based on this principle acquired the name "free electron lasers" (FEL). Because that radiative instability has a narrow spectrum it is not so universal for its use in electron cooling as the Coulomb instabilities, but possibly under conditions the use of radiative mechanism can be profitable.

An additional kind of microwave Coulomb instability which seems easy to realize at non-relativistic energies is the parametric instability of longitudinal plasmas oscillation of the electron beam; it occurs when the plasma parameter of the electron beam, $\omega_{\mathrm{e}}$ modulated with the frequency $\omega \approx 2 \omega_{\mathrm{e}}$. Such a modulation can be realized via modulation of electron beam size or velocity.

Note that the possibilities to provide the correct phase relations and finally the cooling effect are extended with the use of electron plasma oscillations in the input, output, and amplification sections.

In the linear approximation effective friction force increases by a factor $\mathbf{k}=$ $e^{\Lambda L_{c}}$, with respect to (1), where $\Lambda$ is the increment of an instability and $L_{c}$ is the cooling section length. There are, of course, a few limitations on $k$ of a different kind.

An admissible value of $k$ is limited by microwave Schottky-noise of electron beam, which also will increase because of the instability. With "normal" level of noises on the input $k$ should not exceed the mass relation $M / m$, otherwise the heating dominates damping. There are possibilities for suppressing the Schottky-noise effect such as a parametric damping or collision relaxation ${ }^{[7,8]}$; as the result, $k_{a d m}$ can reach the maximum value of order temperature ratio $T_{i} / T_{\text {ell }}$. Note, that the ion temperature can reach a value about $10^{7}-10^{9} \mathrm{~K}$, while the value of electron beam longitudinal temperature is about $\mathrm{e}^{2} \mathrm{n}_{\mathrm{e}}^{1 / 3} \sim 1^{\circ} \mathrm{K}^{[2,3]}$.

An achievable value of $k$ is limited by non-linear saturation of an instability. Taking into account electron and ion beam density fluctuations at the input, we have a limitation on $\mathrm{k}$ as follows:

$$
k_{\max } \sim \min \left\{\mathrm{n}_{\mathrm{e}} \mathrm{R}_{\mathrm{eff}}^{3} ; \Gamma \sqrt{\mathrm{n}_{\mathrm{e}} \mathrm{R}_{\mathrm{eff}}^{3}} ; \mathrm{n}_{\mathrm{e}} \sqrt{\mathrm{R}_{\mathrm{eff}}^{3} / \mathrm{n}_{\mathrm{i}}}\right\},
$$

where $R_{\text {eff }} \sim \rho_{\text {sh }}=\frac{v}{\omega_{\mathrm{e}}}, \Gamma$ is a coefficient of suppression of Schottky-noise amplitude in electron beam and $n_{i}$ is ion concentration (all the parameters in (1) are related to co-moving frame, and we assume an isotropic amplification). Note that $\Gamma$ cannot exceed the value $\Gamma_{\max } \sim \sqrt{\mathrm{e}^{2} \mathrm{n}_{\mathbf{e}} \mathrm{R}_{\text {eff }}^{2} / T_{\text {ell }}}$.

Finally, cooling time is limited by the time of kinematic correlation between particles which arise in a volume of effective interaction:

$$
\tau_{\min } \sim \frac{1}{\gamma} \frac{\mathrm{n}_{\mathrm{i}} \mathrm{R}_{\mathrm{eff}}^{3}}{2 \pi \Delta \mathrm{f}_{0}} \cdot \frac{\mathrm{R}_{\mathrm{eff}}}{\mathrm{R}}
$$


where $\Delta f_{0}$ is the spread of particle revolution frequency, $R$ is machine radius, and $\gamma$ is beam Lorentz-factor. In view of smallnessity of $R_{\text {eff }}\left(R_{e f f} \lesssim a\right.$, where $a$ is beam radius), such a limitation is substantially weaker than in stochastic cooling method, especially at relativistic energies. Considering (2) together with (3), one can find an optimal amplification.

The method considered above combines principles of electron and stochastic cooling and microwave amplification. Such an unification promises to frequently increase the cooling rate and stacking of high-temperature, intensive heavy particle beams. Certainly, for the whole understanding of new possibilities thorough theoretical study is required of all principle properties and other factors of the method.

\section{THE SELF-COOLING OF STRAIGHT BEAMS}

In principle, the electron cooling is capable of cooling a straight low-energetic proton or ion beam within an acceptable length of cooling section; it is effective due to a very low value of the longitudinal electron temperature $\left(\mathrm{T}_{\|}^{\mathrm{e}} \sim 10^{-4} \mathrm{eV}\right)$ and the transverse motion of electrons being bounded because of the magnetic field ${ }^{[2,3]}$. However, in view of the longitudinal heating of electrons by the ions of the beam under cooling, the ratio between the electron's and the ion's current densities must be not less than $\mathrm{T}_{\perp}^{\mathrm{i}} / \mathrm{T}_{\|}^{e}$. Therefore, the use of electron cooling for the ion beams with current density about $\sim 1 \mathrm{~mA} / \mathrm{cm}^{2}$ seems to be problematic.

Now we describe the possibility of using intrabeam scattering of particles for the transverse cooling of a beam, with corresponding heating in the longitudinal direction, during the processes of acceleration and formation of the beam in a straight line ${ }^{[13]}$. There are no external heat energy transfers from the beam; in such a situation, the total beam entropy is not decreased, but there is redistribution between the degrees of freedom of the beam.

We assume axial symmetry of a beam and describe its evolution by variables:

$$
\begin{gathered}
\Gamma_{\perp}=\pi \mathrm{a}^{2} \mathrm{~T}_{\perp}, \quad \Gamma_{\|}=\frac{\gamma v \mathrm{eZ}}{\mathrm{I}} \sqrt{T_{\|}} ; \Gamma=\Gamma_{\perp} \cdot \Gamma_{\|} \\
\mathrm{T}_{\perp}=\frac{\left\langle\left(\Delta \overrightarrow{\mathrm{p}}_{\perp}\right)^{2}\right\rangle}{2 \mathrm{M}} ; \quad \mathrm{T}_{\|}=\frac{\left\langle\left(\Delta \mathrm{p}_{\|}\right)^{2}\right\rangle}{\mathrm{M}} ; \mathrm{I}=\pi \mathrm{a}^{2} \mathrm{n} v Z \mathrm{e},
\end{gathered}
$$

where $\left\langle\left(\Delta \overrightarrow{\mathrm{p}}_{\perp}\right)^{2}\right\rangle$ and $\left\langle\left(\Delta \mathrm{p}_{\|}\right)^{2}\right\rangle$ are transverse and longitudinal dispersion of particle momenta at a given point of space related to a frame moving with an average beam velocity of $v=\left(1-1 / \gamma^{2}\right)^{1 / 2}$, assuming non-relativistic the transverse particle motion, $\mathrm{a}, \mathrm{n}$, and $\tau$ are beam radius, concentration and current, $\mathrm{Ze}$ and $\mathrm{M}$ are particle charge and mass. We also assume for simplicity, that there is no gradient of $T_{\perp}, T_{\|}$and $n$ across the beam. Apparently, we can consider $T_{\perp}$ and $T_{\|}$as effective transverse and longitudinal beam temperatures (related to a given point of the beam). The value $\sqrt{\Gamma_{\perp}}$ is proportional to an 
invariant beam emittance, $\Gamma_{\|}^{-1}$ as an effective beam density in the longitudinal phase space; both of them are dynamical invariants of a beam as an ensemble of particles.

The decreasing of the transverse entropy by collisions between particles then occurs under the condition $\mathrm{T}_{\perp}>\mathrm{T}_{\|}$; this condition can be maintained for a long time by longitudinally stretching the beam during acceleration and by transversely compressing the beam. The increase of the total entropy is small when these processes are performed slow with respect to the process of temperature relaxation. Note that the adiabatic process should start from a state of $T_{\perp}=T_{\|}$, in order to avoid a substantial increase of the entropy during the initial stage of the transverse cooling.

In the adiabatic limit $T_{\perp}=T_{\|}=T$, and $\Gamma$ is still constant under collisions:

$$
\Gamma=\gamma \mathrm{T}^{3 / 2} / \mathrm{n}=\text { const. }
$$

Using (5) and the definitions (4) we get the adiabatic evolution of $\Gamma_{\perp}$ :

$$
\Gamma_{\perp}=\Gamma_{\perp o} \cdot\left[\left(\frac{\mathrm{Ia}}{\gamma v}\right) /\left(\frac{\mathrm{Ia}}{\gamma v}\right)_{0}\right]^{2 / 3} .
$$

The maximum cooling effect would occur when all the process of beam formation and acceleration is performed adiabatically. In this case, the value $v_{0}$ is related to the cathode temperature, $\mathrm{T}_{\mathrm{c}},\left(\mathrm{T}_{\mathrm{c}}=\mathrm{M} v_{0}^{2}\right)$ and $\mathrm{a}_{0}$ is the beam size at the cathode, $a_{c}$. Table 1 gives an illustration of the maximum cooling effect for a heavy particle beam assuming no beam bunching after acceleration.

\begin{tabular}{|l|c|}
\hline \multicolumn{2}{|c|}{ Table 1 } \\
\hline \multicolumn{2}{|c|}{ Maximum Cooling Effect } \\
\hline \multicolumn{2}{|c|}{ Initial beam parameters } \\
\hline Cathode temperature, $\mathrm{eV}$ & 0.1 \\
\hline Beam radius at the cathode, $\mathrm{cm}$ & 0.5 \\
\hline \multicolumn{2}{|c|}{ Parameters after acceleration } \\
\hline Top energy after acceleration, $\mathrm{MeV}$ & 100 \\
\hline Beam radius after acceleration, $\mathrm{cm}$ & 0.02 \\
\hline \multicolumn{2}{|c|}{ Self-cooling effect } \\
\hline Decreasing of beam emittance, times & 100 \\
\hline $\begin{array}{l}\text { Decreasing of transverse phase space } \\
\text { volume, times }\end{array}$ & $10^{4}$ \\
\hline
\end{tabular}


In practice, we cannot use the adiabatic process, but must use the quasiadiabatic process, when change of $\Gamma$ is not zero but small. To formulate a corresponding condition, we define two parameters:

$$
\lambda_{\mathrm{d}}=\frac{\mathrm{Ia}}{\gamma v} /\left(\frac{\mathrm{Ia}}{\gamma v}\right)_{d}^{\prime}, \quad \lambda_{s t}=-\frac{\mathrm{T}_{\perp}-\mathrm{T}_{\|}}{\left(\mathrm{T}_{\perp}-\mathrm{T}_{\|}\right)_{\mathbf{s t}}^{\prime}}
$$

where the symbol " $\mathrm{d}$ " denotes change of parameters in an external field without collisions, and "st" means change under collisions. To calculate parameter $\lambda_{\text {st }}$ near the equilibrium state, we use the Landay collision integral ${ }^{[14]}$ at the model of Gaussian distribution in temperatures $T_{\perp}$ and $T_{\|}$, then we get

$$
\lambda_{\mathrm{st}}=\frac{5}{8} \sqrt{\frac{\mathrm{M}}{\pi}} \cdot \frac{\gamma v}{(\mathrm{Ze})^{4} \mathrm{~L}} \Gamma
$$

where $\mathrm{L}$ is the Coulomb parameter $\mathrm{L}=\frac{1}{2} \ell \mathrm{n}\left[\gamma \mathrm{T}^{3} / 4 \pi \mathrm{n}(\mathrm{Ze})^{6}\right]$ with an order of value about 3-5.

At $\lambda_{\mathbf{d}} \gg \lambda_{\mathrm{st}}$ we can get the equation describing $\Gamma$ increase:

$$
\Gamma^{\prime} \approx\left(\lambda_{\mathrm{st}} / 3 \lambda_{\mathrm{d}}^{2}\right) \Gamma
$$

Solving this equation, one can establish the boundaries of stability of quasiadiabatic process and calculate non-adiabatic effects.

When accelerating an actual beam, the adiabatic condition cannot be satisfied in the region near the cathode, because the characteristic time of acceleration there is about equal to the inverse plasma parameter $\omega_{\mathrm{p}}^{-1}=\sqrt{\mathrm{M} / 4 \pi \mathrm{n}(\mathrm{Ze})^{2}}$, which is small in comparison to the temperature relaxation time $\lambda_{\text {st }} / \mathrm{u}$. With the acceleration, the longitudinal temperature goes down very fast, and one must take into account intra-beam scattering which can limit its decrease, i.e. the longitudinal entropy will increase with collisions between particles. After a distance of about $a_{c}$ from the cathode, we can equalize the transverse and longitudinal temperatures by having the transverse expansion of the beam and, if necessary, by deacceleration of the beam. In this state, we obtain an intermediate energy $\mathrm{W}_{0}$ such that $\mathrm{T}_{\mathrm{c}} \ll \mathrm{W}_{0} \ll \mathrm{W}_{\max }$, with initial (maximum) radius $\mathrm{a}_{0}$ and initial value of relaxation parameter $\lambda_{\text {st }}$. With these parameters, we can start the quasiadiabatic cooling process. In view of the presence of the non-adiabatic stage at the beginning of the beam evolution, the self-cooling effect will be less than potentially possible as was presented in Table 1 (see Table 2 for a case $|\mathrm{Z}|=1)$. 
In addition, we should note the following conditions for the beam dynamics in focusing and accelerating:

1. Axially symmetrical electrodes and solenoidal magnetic field can be used in order to keep an intensive low energetic beam from repulsion by the space charge.

2. The current distribution at the cathode and the accelerating electric field should have axial symmetry.

3. Electric and magnetic fields have to be matched in the region of the beam injection into the solenoid, in order to avoid radial beam excitation inside the solenoid, i.e., to reach the Brillouin's beam state ${ }^{[15]}$.

\begin{tabular}{|l|c|}
\hline \multicolumn{2}{|c|}{ Table 2} \\
\hline \multicolumn{2}{|c|}{ Self-cooling of an Actual Beam } \\
\hline Beam current $\mathrm{I}, \mathrm{A}$ & 1 \\
\hline Beam radius at cathode $\mathrm{a}_{\mathrm{c}}, \mathrm{cm}$ & 0.5 \\
\hline Cathode temperature $\mathrm{T}_{\mathrm{c}}, \mathrm{eV}$ & 0.1 \\
\hline Anode voltage $\mathrm{V}_{\mathrm{A}}, \mathrm{kV}$ & 10 \\
\hline Longitudinal length of expansion section $\mathrm{cm}$ & 6 \\
\hline Beam radius after expansion $\mathrm{a}_{0}, \mathrm{~cm}$ & 3 \\
\hline Initial energy of the adiabatic process $\mathrm{W}_{0}, \mathrm{keV}$ & 10 \\
\hline Initial relaxation length $\lambda_{0}, \mathrm{~m}$ & 0.3 \\
\hline Final energy $\mathrm{W}_{\mathrm{f}}, \mathrm{MeV}$ & 100 \\
\hline Maximum value of solenoidal field $\mathrm{B}_{\mathrm{f}}$, Tesla & 10 \\
\hline Final beam radius in the solenoid $\mathrm{a}_{\mathrm{f}}, \mathrm{cm}$ & 0.02 \\
\hline Final relaxation length $\lambda_{\mathrm{f}}, \mathrm{m}$ & 15 \\
\hline Cooling effect on beam emittance, times & 25 \\
\hline Cooling effect on beam brightness, times & 600 \\
\hline
\end{tabular}

Note that the considered method of cooling is related to the Boersch effect ${ }^{[15]}$, which is longitudinal heating of an accelerated beam due to intrabeam scattering at $T_{\perp}>T_{\|}$. Our observation is that one can deeply cool a beam transversally if accelerating and compressing it adiabatically with respect to temperature relaxation process. 


\section{REFERENCES}

1. G. I. Budker, At. En. (Sov. ) 33, 346 (1967).

2. Ya. S. Derbenev and A. N. Skrinsky, Sov. Phys. Rev. 1, 165 (1981).

3. V. V. Parkhomchuk, A. N. Skrinsky, Preprint IYaF AN SSSR No. 90-102 (1990).

4. S. van der Meer, CERN/ISR-RF/72-46 (1972).

5. Ya. S. Derbenev and S. A. Kheifets, Part. Accel. 9, 237 (1979).

6. S. Schröder, et al., Phys. Rev. Lett. 64, 2901 (1990).

7. Ya. S. Derbenev, Proceedings of the $7^{\text {th }}$ National Acc. Conference, V. 1, p. 269 (Dubna 1981).

8. Ya. S. Derbenev, UM HE 91-28, Ann Arbor, Michigan (1991).

9. A. M. Kondratenko and E. L. Saldin, Docl. of Ac. Sc. (Sov.) 249, 843 (1979).

10. N. M. Kroll and W. A. McMillan, Phys. Rev. A17, 300 (1978).

11. A. V. Burov, Ya. S. Derbenev, Preprint TYaF AN SSSR No. 81-33 (1981).

12. A. S. Artamonov and N. I. Inozemtsev, Sov. J. Commun. Tech. Electronics (USA), 34, 52 (1989).

13. Ya. S. Derbenev, UM HE 91-13, Ann Arbor, Michigan (1991).

14. L. D. Landay, Zh. Eskp. Teor. Fiz. 7, 203 (1937).

15. J. D. Lawson, "The Physics of Charged Particle Beams" (Clarendon Press, Oxford 1988). 